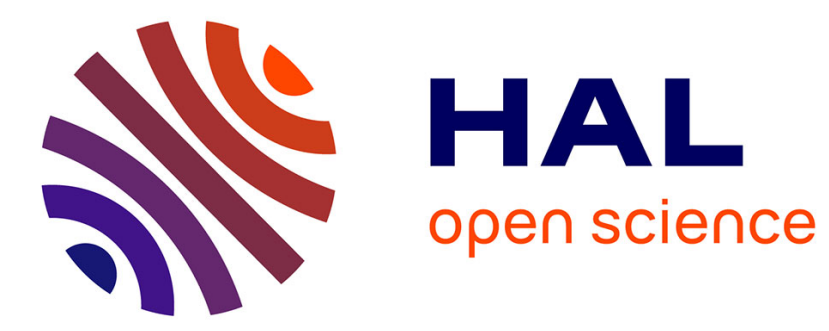

\title{
Traffic Control via Platoons of Intelligent Vehicles for Saving Fuel Consumption in Freeway Systems
}

\author{
Giulia Piacentini, Paola Goatin, Antonella Ferrara
}

\section{To cite this version:}

Giulia Piacentini, Paola Goatin, Antonella Ferrara. Traffic Control via Platoons of Intelligent Vehicles for Saving Fuel Consumption in Freeway Systems. IEEE Control Systems Letters, 2020, 10.1109/LCSYS.2020.3004754 . hal-02883799

\section{HAL Id: hal-02883799 \\ https://hal.science/hal-02883799}

Submitted on 29 Jun 2020

HAL is a multi-disciplinary open access archive for the deposit and dissemination of scientific research documents, whether they are published or not. The documents may come from teaching and research institutions in France or abroad, or from public or private research centers.
L'archive ouverte pluridisciplinaire HAL, est destinée au dépôt et à la diffusion de documents scientifiques de niveau recherche, publiés ou non, émanant des établissements d'enseignement et de recherche français ou étrangers, des laboratoires publics ou privés. 


\title{
Traffic Control via Platoons of Intelligent Vehicles for Saving Fuel Consumption in Freeway Systems
}

\author{
G. Piacentini ${ }^{1}$, P. Goatin ${ }^{2}$, A. Ferrara ${ }^{1}$
}

\begin{abstract}
In this paper a coupled PDE-ODE model describing the interaction between the bulk traffic flow and a platoon of connected vehicles is adopted to develop a control action aiming at reducing the fuel consumption of the overall traffic flow. The platoon is modeled as a capacity restriction acting on the surrounding traffic. The trajectory of the initial and final points of the platoon are optimized by means of a model predictive control strategy, acting on the speeds of the front-end and backend of the platoon, thus resulting in controlling both the speed and the length of the platoon. The approach is assessed in simulations.
\end{abstract}

Index Terms-Traffic control, Autonomous vehicles, Optimal control

\section{INTRODUCTION}

$\mathbf{N}$ OWADAYS the level of connectivity, automation and autonomy that the automotive industry is achieving is completely transforming the transportation sector impacting also the social-economical aspects. Vehicle Automation and Communication Systems (VACSs) improve driving convenience due to support tools, which assist drivers in vehicle control to reduce the stress of driving. They also provide information to users as well as increase road safety, thus leading to improvements in terms of accidents avoidance and mitigation. In addition to this, VACSs pave the way for a better utilization of resources, aiming at improving traffic and environmental efficiency, considering that vehicular traffic is one of the main sources of emissions and pollution [1]. A more efficient road utilization can be envisaged exploiting intelligent vehicles as actuators to reduce traffic congestion, a phenomenon that daily occurs due to the increasing traffic demand. The presence of congestion on highways tends to modify the driving behaviour introducing the need of a higher number of speedups, slowdowns, stops and starts, which increase fuel consumption and emissions. This strongly degrades ambient air quality and increases people health risks [2]. An effective management of highways can lead to important benefits as reduction of travel times for drivers, improved safety and reduced emissions. Several traffic management strategies have been developed in the last decades proving to be effective in enhancing highway conditions. Traditional methods include variable speed limits [3] and ramp metering [4], but they have the drawback of requiring specific infrastructure. The recent

\footnotetext{
${ }^{1}$ G. Piacentini, A. Ferrara are with the Department of Electrical, Computer and Biomedical Engineering, University of Pavia, Italy. giulia.piacentini02euniversitadipavia.it, antonella.ferrara@unipv.it

${ }^{2} \mathrm{P}$. Goatin is with Université Côte d'Azur, Inria, CNRS, LJAD, France paola.goatineinria.fr
}

advances in information and communications technologies, connectivity and automation, introduce the possibility for new traffic management techniques [5]. In this perspective, the idea of controlling a small number of intelligent vehicles acting as actuators to influence the surrounding human-driven traffic flow has been recently investigated. Field experiments have demonstrated that the control of a small number of automated vehicles can help to dissipate stop-and-go waves deriving from human driving behavior, thus harmonizing the traffic and reducing emissions [6].

Several modeling approaches have been proposed to model the interaction between the normal flow and automated vehicles. While some research works move in the direction of microscopic models [7], macroscopic models are often preferred for control applications due to their lower computational complexity. Some extensions to traditional macroscopic models have been studied in order to model the presence of intelligent vehicles moving in the neighbouring human-driven traffic flow. Some approaches have considered automated vehicles as they were moving bottlenecks that, although they are usually seen as impediments to efficient traffic flow, can be actually controlled to advantageously influence the surrounding traffic. Among others, in [8], [9], models consisting of a scalar conservation law for the traffic evolution and of an ODE accounting for the trajectory of moving bottlenecks have been proposed. In [10], the model presented in [8] is used to describe the traffic flow and the speed of the moving bottleneck is assumed as control variable to reduce the fuel consumption of the overall traffic flow. In [11] moving bottlenecks are controlled to solve congestion on the highway in a Cell Transmission Model (CTM) framework [12]. In addition to singularly control each Connected Automated Vehicle (CAV), their formations, as platooning, can be studied to improve traffic control efficiency. Opportunities for cooperative driving can indeed introduce the possibility for energy efficiency of groups of vehicles moving in a coordinated manner, and this can have a good impact on the surrounding mixed flow [13]. Eco-driving and energy efficiency considerations also suggest to adopt platooning on highways and this could be exploited to increase the throughput, if properly controlled [14],[15]. In [16], [17], the authors proposed an extension of the CTM accounting for the presence of multiple platoons of CAVs as constraints on the free-flow speed of the cells occupied by the platoon. Their speeds were controlled by means of a Model Predictive Control (MPC) to reduce travel times of drivers. The PDE-ODE model presented in [8] considering a punctual moving bottleneck has been recently extended in [18] to capture platoons of vehicles having a physical length that 


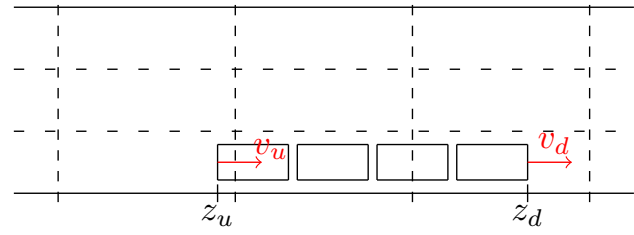

Figure 1: Scheme of platoon on the highway.

can change in time, depending on the number of vehicles composing it and their spacing, so that the length can here be taken as an additional control variable. The main contribution of this paper is the design of a multi-variable model predictive control to regulate the speeds of both end-points of the platoon (therefore controlling its speed and length) with the aim of reducing congestion and fuel consumption of the overall traffic flow. Therefore, in the present work, the focus is on the control of the bulk traffic flow, while the platoon is conceived as an actuator for the control action and its internal dynamics is not taken into account. Specifically, an high-level control optimizes a performance index related with the traffic flow to obtain the optimal length and speed for the platoon. A lower level control will then receive input information regarding the reference length and the speed for the platoon and will implement a proper spacing policy spacing to guarantee its formation. In section II the adopted model for the platoon is outlined, section III describes the model predictive control action that is applied to the system. In section IV simulation results are reported and a comparison between the results obtained in [10] is made.

\section{THE MODEL}

The present paper deals with a macroscopic first-order model, which describes a platoon of vehicles moving on highway, and its impact on the neighbouring traffic. We consider an highway with a given number of lanes, where the general evolution of the traffic flow is captured by means of a hyperbolic Partial Differential Equation (PDE), according to the standard Lighthill-Whitam-Richards (LWR) model [19], [20]. Two Ordinary Differential Equations (ODEs) track the trajectories of the front and back endpoints of the platoon. The capacity of the highway is reduced in correspondence of the platoon location. We indicate with $\rho=\rho(x, t)$ the density of vehicles and with $F=F(t, x, \rho)$ the flow function, while $v=v(\rho)$ is the average speed of the flow. The downstream and upstream endpoints of the platoon are denoted by $z_{d}=z_{d}(t)$ and $z_{u}=z_{u}(t)$, as depicted in Fig. 1. We indicate with $i_{d}=i_{d}(t)$ and $i_{u}=i_{u}(t)$ the cells in which $z_{d}=z_{d}(t)$ and $z_{u}=z_{u}(t)$ lie respectively. The portion of highway interested by the presence of the platoon, i.e. the interval $\left[z_{u}(t), z_{d}(t)\right]$, have reduced capacity due to the lanes occupied by the platoon. The model is then described by the following equations

$$
\begin{array}{lr}
\partial_{t} \rho+\partial_{x} F(t, x, \rho)=0, & (t, x) \in \mathbb{R}^{+} \times \mathbb{R}, \\
\rho(0, x)=\rho_{0}(x), & x \in \mathbb{R}, \\
\dot{z}_{u}(t)=v_{u}\left(t, \rho\left(t, z_{u}(t)+\right)\right), & t \in \mathbb{R}^{+}, \\
z_{u}(0)=z_{u}^{0}, &
\end{array}
$$

$$
\begin{aligned}
& \dot{z}_{d}(t)=v_{d}\left(t, \rho\left(t, z_{d}(t)+\right)\right), \quad t \in \mathbb{R}^{+}, \\
& z_{d}(0)=z_{d}^{0}
\end{aligned}
$$

where the flow function $F$ is given by

$$
F(t, x, \rho):= \begin{cases}f(\rho) & \text { if } x \notin\left[z_{u}(t), z_{d}(t)\right] \\ f_{\alpha}(\rho):=\alpha f(\rho / \alpha) & \text { if } x \in\left[z_{u}(t), z_{d}(t)\right]\end{cases}
$$

Since the platoon acts as a flux constraint on the interval $\left[z_{u}(t), z_{d}(t)\right], \alpha \in[0,1]$ represents the capacity reduction rate, i.e. the ratio of lanes not occupied by the platoon, and at the platoon location the reduced flow $f_{\alpha}$ is considered. Considering the linear speed-density relation $v(\rho)=V\left(1-\frac{\rho}{\rho_{\max }}\right)$ we get

$$
\begin{gathered}
f(\rho)=V \rho\left(1-\frac{\rho}{\rho_{\max }}\right), \\
f_{\alpha}(\rho)=V \rho\left(1-\frac{\rho}{\alpha \rho_{\max }}\right),
\end{gathered}
$$

where $V$ is the maximum speed of the traffic flow and $\rho_{\max }$ the maximum value of the density. The trends of the normal flow $f$ and the reduced flow $f_{\alpha}$ are depicted in Fig. 2. Equations (1c) and (1e) describe the trajectories of the initial and final points of the platoon. The speed of the downstream point is given by

$$
v_{d}(t, \rho):=\min \left\{V_{d}(t), v(\rho)\right\}
$$

where $V_{d}(t) \in[0, V]$ is the maximal speeds for $z_{d}(t)$. Eq. (5) means that the platoon has to adapt its speed to the downstream traffic velocity, i.e. the platoon does not have any impact on the traffic if the control speed is higher than the downstream traffic speed. Concerning the speed of the upstream point, we set

$$
v_{u}(t, \rho):=\max \left\{V_{u}(t),-f_{\alpha}(\rho) /\left(\rho_{\max }-\rho\right)\right\},
$$

where $V_{u}(t) \in[-V, V]$ is the maximal speeds for $z_{u}$. The technical constraint (6) comes from an admissibility condition on the feasibility of the corresponding Riemann problems, see [18]. The speed of the upstream point $v_{u}$ is allowed to assume a negative value if new vehicles can join the platoon, thus moving the end point backwards. The length of the platoon $L(k)=z_{d}(k)-z_{u}(k)$ varies in time depending on the speed of the front and back end-points of the platoon, specifically

$$
L(k+1)=L(k)+\left(v_{d}(k)-v_{u}(k)\right) \Delta t .
$$

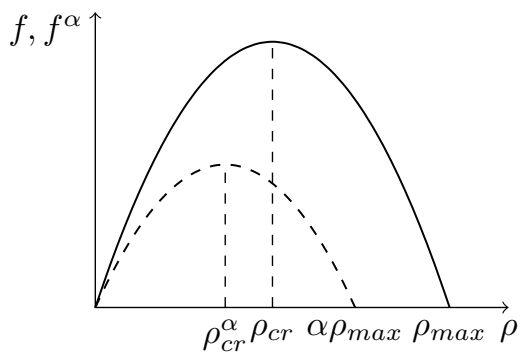

Figure 2: Normal and reduced flow. 
The platoon length can be modified by variations in the spacing between vehicles and if the number of cars joining the platoon changes.

To solve the space-discontinuous conservation law (1a), the model is discretized in both time and space, thus sampling with time interval $\Delta t$ and dividing the stretch of highway in $N$ cells $i=1, \ldots, N$ of length $\Delta x$. The discretization steps are chosen according to the Courant-Friedrichs-Lewy (CFL) condition $2 V \Delta t \leq \Delta x$. The upstream and downstream interfaces of a generic cell $i$ are indicated as $x_{i-1 / 2}, x_{i+1 / 2}$. The minimum allowable platoon length and the length cell $\Delta x$ are chosen so that $z_{d}(t)$ and $z_{u}(t)$ are never located in the same cell. At each time step $k$ the state equation updating the density in each cell $i$ is given as

$$
\rho_{i}(k+1)=\rho_{i}(k)+\frac{\Delta t}{\Delta x}\left(F_{i-1}(k)-F_{i}(k)\right)
$$

where $F_{i-1}$ is the flow between cell $i-1$ and cell $i$, that is computed by means of the standard supply-demand paradigm [12] for all the cells, except the ones in which the platoon interfaces lie. Since a reduction of the flow in the platoon cells appears, reduced demand and supply are consequently defined in that areas. Specifically, the demand and supply of a generic cell $i$ are computed as

$$
\begin{aligned}
& D_{i}(k)= \begin{cases}f\left(\rho_{i}(k)\right) & \text { if } \rho_{i}(k)<\rho_{c r}, \\
f^{\max } & \text { if } \rho_{i}(k) \geq \rho_{c r},\end{cases} \\
& S_{i}(k)= \begin{cases}f^{\max } & \text { if } \rho_{i}(k)<\rho_{c r}, \\
f\left(\rho_{i}(k)\right) & \text { if } \rho_{i}(k) \geq \rho_{c r},\end{cases}
\end{aligned}
$$

for $i<i_{u}$ or $i>i_{d}$, i.e. where there is not the platoon. Above, $\rho_{c r}=0.5 \rho_{\max }$ denotes the critical density, and $f^{\max }=f\left(\rho_{\max }\right)$ is the maximal flux on the road.

Similarly, for $i_{u}<i<i_{d}$, the demand and supply are computed considering the reduced flux:

$$
\begin{gathered}
D_{i}^{\alpha}(k)= \begin{cases}f_{\alpha}\left(\rho_{i}(k)\right) & \text { if } \rho_{i}(k)<\rho_{c r}^{\alpha}, \\
f_{\alpha}^{\max } & \text { if } \rho_{i}(k) \geq \rho_{c r}^{\alpha},\end{cases} \\
S_{i}^{\alpha}(k) \begin{cases}f_{\alpha}^{\max } & \text { if } \rho_{i}(k)<\rho_{c r}^{\alpha}, \\
f_{\alpha}\left(\rho_{i}(k)\right) & \text { if } \rho_{i}(k) \geq \rho_{c r}^{\alpha},\end{cases}
\end{gathered}
$$

where $\rho_{c r}^{\alpha}=0.5 \alpha \rho_{\max }$ and $f_{\alpha}^{\max }=f_{\alpha}\left(\rho_{c r}^{\alpha}\right)$.

Except for the cells $i_{d}$ and $i_{u}$ accommodating the end-points of the platoon, the flow between two generic cells is given by the minimum between the demand of the upstream cell and the supply of the downstream cell:

$$
\begin{aligned}
& F_{i}(k)=\min \left\{D_{i-1}(k), S_{i}(k)\right\} \text { for } i \leq i_{u}-2, i \geq i_{d}+1, \\
& F_{i}(k)=\min \left\{D_{i-1}^{\alpha}(k), S_{i}^{\alpha}(k)\right\} \text { for } i \geq i_{u}+1, i \leq i_{d}-2 .
\end{aligned}
$$

In correspondence of the initial and final points of the platoon, a discontinuity in the flow arises and a solution to the scalar conservation law with moving discontinuities needs to be studied. A numerical scheme able to precisely capture the density discontinuities has been proposed in [18], where a conservative reconstruction strategy is adopted. For both the upstream and downstream endpoints of the platoon, we consider the discontinuities moving with speeds $v_{u}(k)$ and $v_{d}(k)$. More precisely, the positions of the end-points of the platoon are updated as

$$
\begin{aligned}
& z_{d}(k+1)=z_{d}(k)+\min \left\{V_{d}(k), v\left(\rho_{i_{d}+1}(k)\right\} \Delta t,\right. \\
& z_{u}(k+1)=z_{u}(k)+V_{u}(k) \Delta t .
\end{aligned}
$$

We study the solution to the problem at the two interfaces separately. Let us focus on the cell $i_{d}(k)$ in which the downstream front-end point $z_{d}(k)$ is located at time step $k$. We expect a density discontinuity to appear in the cell and at each time step we consider the Riemann-type initial datum $\rho_{l}=\rho_{i_{d}-1}(k)$ and $\rho_{r}=\rho_{i_{d}+1}(k)$. The Riemann problem is then solved as detailed in [18] and we obtain the two solution density values $\hat{\rho}_{\alpha}$ and $\check{\rho}$, that correspond to the values of density that appear in cell $i_{d}$ upstream and downstream the position $z_{d}(k)$. The density $\rho_{i_{d}}(k)$ can be then replaced by a convex combination of the two values by defining $d_{i_{d}}$ such that

$$
\hat{\rho}_{\alpha} d_{i_{d}}+\check{\rho}\left(1-d_{i_{d}}\right)=\rho_{i_{d}}(k), \quad \text { i.e. } \quad d_{i_{d}}=\frac{\rho_{i_{d}}(k)-\check{\rho}}{\hat{\rho}_{\alpha}-\check{\rho}} .
$$

The reconstructed discontinuity is located at the position $\bar{x}_{i_{d}}=x_{i_{d}-1 / 2}+d_{i_{d}} \Delta x$ and the numerical flux at the interface $x_{i_{d}+1 / 2}$ is then reconstructed as

$$
\begin{aligned}
\Delta t F_{i_{d}}(k)=\min \left\{\Delta t_{i_{d}}, \Delta t\right\} & f(\check{\rho}) \\
+ & \max \left\{\Delta t-\Delta t_{i_{d}}, 0\right\} f_{\alpha}\left(\hat{\rho}_{\alpha}\right),
\end{aligned}
$$

where

$$
\Delta t_{i_{d}}=\frac{1-d_{i_{d}}}{\min \left\{V_{d}(k), v\left(\rho_{i_{d}+1}(k)\right)\right\}} \Delta x
$$

represents the time that the discontinuity needs to reach the downstream interface $x_{i_{d}+1 / 2}$, as depicted in Fig. 3. On the other end, we set

$$
F_{i_{d-1}}(k)=\min \left\{D^{\alpha}\left(\rho_{i_{d-1}}(k)\right), S^{\alpha}\left(\hat{\rho}_{\alpha}(k)\right)\right\}
$$

The same identical approach is applied to the back-end of the platoon.

\section{CONTROL APPROACH}

The aim of the present work is to design a controller that minimizes the fuel consumption of the overall traffic flow controlling the maximum speed of the downstream and upstream end-points of the platoon, $V_{d}$ and $V_{u}$ respectively. This results in controlling both the speed and the length of the platoon, due to Eq. (7). The fuel consumption is computed by means of the model presented in [10], [21]. Variations in vehicle fuel consumption are related to the average speed, with higher consumption at very low and very high speeds.

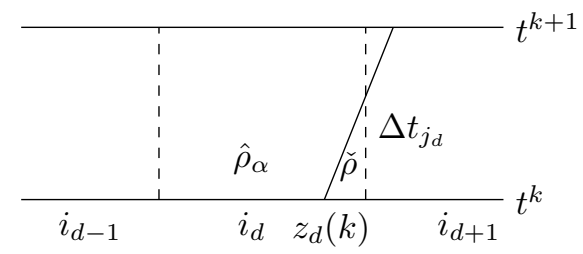

Figure 3: Representation of the flux reconstruction at the downstream endpoint of the platoon. 
Following [21], a curve averaged on the characteristics of different cars is computed and approximated by a sixth order polynomial $K(v)$ :

$$
\begin{aligned}
& K(v)=5.7 \cdot 10^{-12} \cdot v^{6}-3.6 \cdot 10^{-9} \cdot v^{5}+7.6 \cdot 10^{-7} \cdot v^{4}- \\
& \quad-6.1 \cdot 10^{-5} \cdot v^{3}+1.9 \cdot 10^{-3} \cdot v^{2}+1.6 \cdot 10^{-2} \cdot v+0.99 .
\end{aligned}
$$

Above, $K(v)$ is expressed in [Liters $/ h r]$ and $v$, the traffic speed, in $[\mathrm{km} / \mathrm{hr}]$. The function $K(v)$ is re-parametrized in terms of density following the linear speed-density relationship to obtain the fuel consumption rate of vehicles as a function of the density at the vehicle position, denoted as

$$
F C(\rho)=K(v(\rho)) .
$$

The overall fuel consumption rate $T F C(\rho)$ is so computed by

$$
T F C(\rho)=\rho F C(\rho) .
$$

The control problem is solved by means of a $M P C$ approach. $V_{d}$ and $V_{u}$ are assumed as control variables and we denote as $u=\left[V_{d}, V_{u}\right]$ the vector of the control inputs. We indicate as $K_{p}$ the number of time steps of the prediction horizon. At a fixed time step $k$, given the current initial state $\rho(k)$, we compute an optimal control sequence $\underline{u}(h)=\left[\underline{V_{d}}(h) \underline{V_{u}}(h)\right]$, $h=k, \ldots, k+K_{p}$, that minimizes the given objective function. The control $\underline{u}$ is constrained to be piece-wise constant on subintervals of size $\Delta t K_{p} / \ell$, for some $\ell \in \mathbb{N}$ in order to reduce the computational complexity deriving from the optimization. At $t=k \Delta t$, only the first input $\underline{u}(k)=\left[\underline{V_{d}}(k) \underline{V_{u}}(k)\right]$ of the optimal control sequence is applied to the real system on the time interval $\left[t, t+\Delta t K_{p} / \ell\right]$. At the following iteration, based on the new current information, the optimization is repeated shifting the horizon. Since we are dealing with a non-linear system, the problem can only be solved numerically, by means of iterative optimization algorithms requiring, at each iteration, to compute approximate solutions of system (1). At the $k$-th iteration, the optimal input speeds are solution of the following constrained multi-variable optimization problem

$$
\min _{\underline{u}} \sum_{h=k}^{k+K_{p}} \sum_{i=1}^{N} T F C\left(\rho_{i}(h)\right) \Delta x \Delta t,
$$

subject to the model dynamics (1), (5), (6) and to the following constraints

$$
\begin{aligned}
& L_{\min } \leq L(h) \leq L_{\max } \\
& V_{d}^{\min } \leq V_{d}(h) \leq V^{\max }, \\
& \left|V_{d}(h)-V_{u}(h)\right| \leq c .
\end{aligned}
$$

for $h=k, \ldots, k+K_{p}$.

The first constraint (15a) limits the minimum and the maximum length that the platoon can assume. The value of $L_{\text {min }}$ depends on safety distance considerations, while $L_{\max }$ is related to fuel efficiency evaluations of vehicles in the platoon, since an excessive distancing between them would reduce the advantage for vehicles to stay in the platoon. Constraint (15b) prevents the platoon to assume too low speeds on the highway. The constraint expressed by Eq. (15c) limits the rate at which the platoon can change its length, $c$ is a suitable threshold

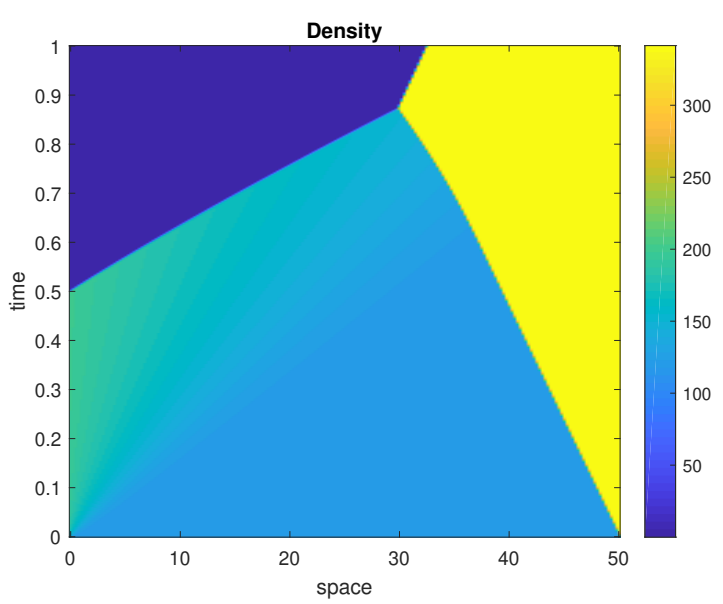

(a) no control

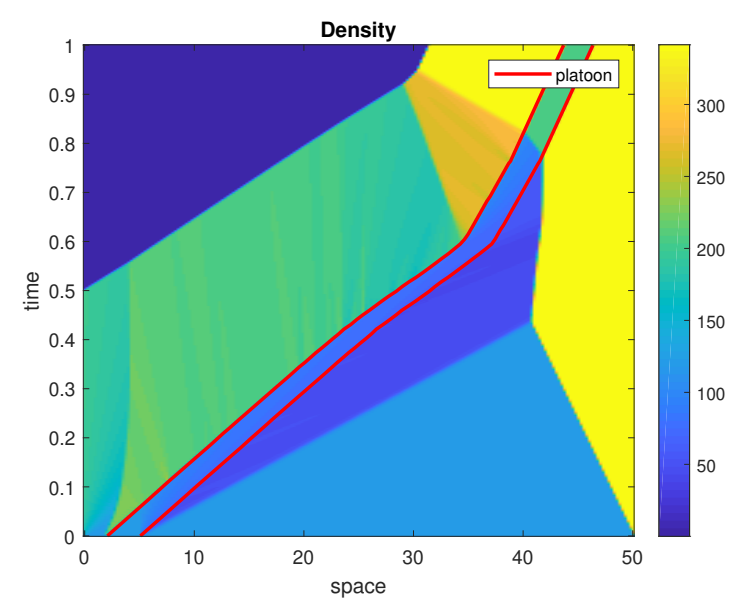

(b) MPC control

Figure 4: Density trend

chosen in order to avoid accordion effects in the variation of length of the platoon. The parameter $c$ has to be identified based on several conditions, as the type of the highway (number of lanes, slope, etc.) and categories of vehicles in the platoon, as cars, trucks etc.

\section{Simulation RESULTS}

The approach is studied by means of simulations considering an highway stretch without ramps. The road segment is subdivided in 200 cells of length $250[\mathrm{~m}]$, where the traffic flow is described by means of the quadratic fundamental diagram (3) with maximum speed $V=140[\mathrm{~km} / \mathrm{h}]$, maximum density $\rho_{\max }=400[\mathrm{veh} / \mathrm{km}]$ and capacity $f^{\max }=$ $14000[v e h / h]$. The sampling time, chosen according to the CFL condition, is $\Delta t=5.76[\mathrm{sec}]$, the total simulation time is one hour. A platoon of initial length of $3[\mathrm{~km}]$ is considered, located at position $z_{d}\left(t_{0}\right)=4[\mathrm{~km}]$ with initial speeds $v_{d}\left(t_{0}\right)=v_{u}\left(t_{0}\right)=80[\mathrm{~km} / \mathrm{h}]$. The value of the capacity reduction rate $\alpha$ is 0.6 . The incoming flow is equal to the capacity $f^{\max }$ for the first half of the simulation and it becomes null in the second half. As in [10], the outflow from the last cell is reduced to half of the capacity, simulating the presence of a fixed bottleneck inducing congestion at the end 


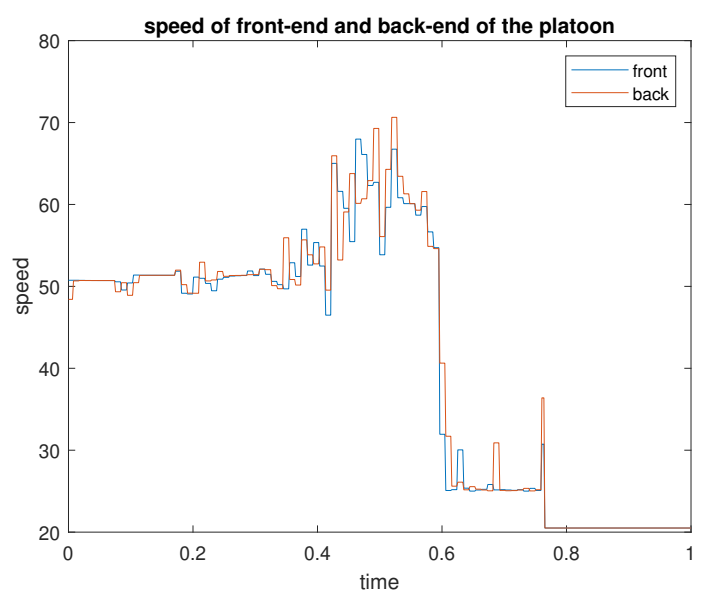

(a) Control speeds

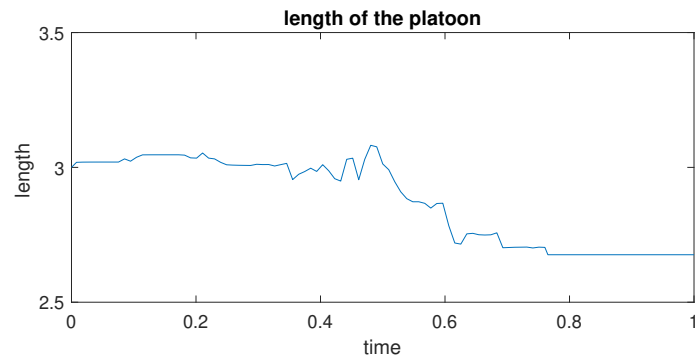

(b) Platoon length

Figure 5: Speed of the front-end and back-end of the platoon and length varying in time.

of the highway. For the MPC, a prediction horizon of 40 time steps is chosen, but, in order to reduce the computational burden deriving from the solution of the non-linear optimization problem, each value of the speed is kept constant for 5 time steps. For the constraints of Eq. (15), the platoon minimal and maximum allowable lengths are $L_{\min }=2[\mathrm{~km}]$ and $L_{\max }=4[\mathrm{~km}], V_{d}^{\min }=40[\mathrm{~km} / \mathrm{h}]$, and $c=30[\mathrm{~km} / \mathrm{h}]$. The non-linear optimization is solved by means of the fmincon function of the MATLAB optimization toolbox. In Fig. 4, the spatio-temporal evolutions of the density are reported for both the controlled and the uncontrolled scenario, while the red lines indicate the trajectory of the platoon. In the case without control (Fig. 4(a)), cars travel at their maximum allowable speed until they reach the congestion due to the bottleneck. Here the speed of vehicles drops down, since they reach a congested region with high density values. This behaviour causes an high fuel consumption, since vehicles travel always either at very high or very low speed, which are the worst situations in terms of fuel consumption. In Fig. 4(b) the proposed control is applied. The controller tends to slow down the platoon and to modify its length in order to prevent vehicles to travel too fast towards the congestion, where they should abruptly brake. In this way, the traffic near the platoon is harmonized and vehicles have a moderate speed that is beneficial for reducing the fuel consumption. The computed optimal speeds applied to the platoon and its length are reported in Fig. 5. In the last part of the simulation, the platoon enters the area with high congestion and its speed is not controlled anymore but it

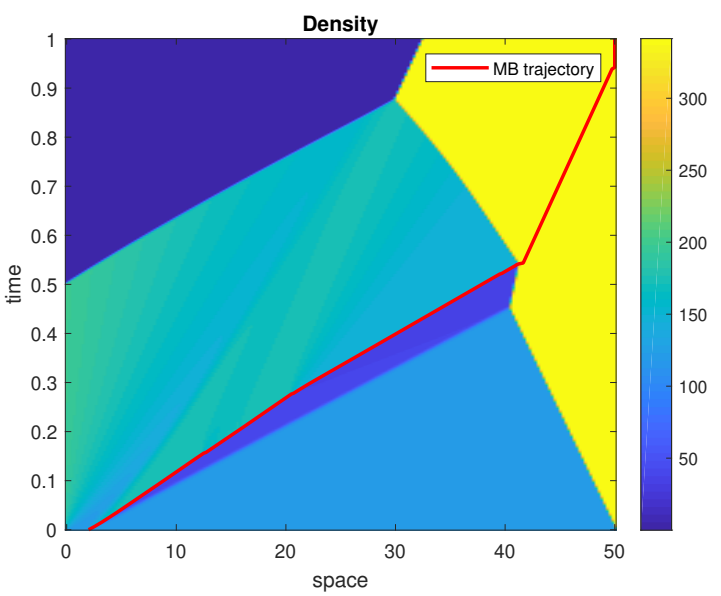

Figure 6: Density trend in the simple moving bottleneck control of [10]

adapts to the speed of the downstream traffic. The value of the TFC obtained with this control strategy is reported in Table I. A reduction of the Total Fuel Consumption (14) of the $2.6 \%$ is obtained by applying the proposed approach, leading the fuel consumption of the overall traffic flow from 27629 liters to 26903 liters, with a saving of 726 liters of fuel. Although this reduction may seem small, it represents a significant reduction of emissions that is beneficial for the environment. It is important to consider that the improvement is here obtained by controlling only one platoon. Considering a scenario in which CAVs will be present, this control could be applied without adding any additional infrastructure and therefore at low cost. In this perspective, several platoons of vehicles may be formed and controlled to increase the effectiveness of the control. Moreover, in this work we do not focus on individual vehicles traveling in the platoon. As they are concerned, it could be possible to observe a reduction in the consumed fuel [22], since a more harmonized driving behaviour emerges and the aerodynamics of vehicles in the platoon is improved at reduced interdistances. Also, this aspect would be more accentuated if the controlled vehicles were autonomous trucks [23], [24]. A comparison with the control action designed in our previous work [10] can be done. The main difference with [10] is that it considers a model describing the impact of a single vehicle acting as a moving bottleneck, i.e. a punctual capacity constraint without physical dimension. In that case, only the speed of the moving bottleneck was controlled. In the present work, we have an additional degree of freedom given by the length of the platoon and we expect a more effective control action. The results obtained in the same simulation scenario are here compared. The trend of the density in the moving bottleneck control situation is reported in Fig. 6, while the fuel consumption performances are compared in Table I, showing that the platoon control is more effective in reducing the fuel consumption with respect to a single vehicle.

\section{CONClusion}

In this work we apply a new macroscopic model able to precisely capture the presence of platoons of CAVs in freeway systems. We design a control strategy to reduce the fuel 
Table I: Comparison between cost functionals.

\begin{tabular}{lcc}
\hline & TFC & reduction $\%$ \\
\hline No control & $2.7629 \mathrm{e}+04$ & $0 \%$ \\
\hline MB control & $2.6988 \mathrm{e}+04$ & $2.29 \%$ \\
\hline Platoon control & $2.6903 \mathrm{e}+04$ & $2.63 \%$ \\
\hline
\end{tabular}

consumption of the overall traffic flow. The speeds of the two endpoints of the platoon are assumed as control variables by using a model predictive control approach, thus regulating both the speed and length of the platoon to minimize the fuel consumption, and consequently reducing emissions and pollution. The approach has been studied in simulations, assessing the validity of the approach. Future works include coordinated control of multiple platoons to increase the effectiveness of the control action.

\section{REFERENCES}

[1] C. Diakaki, M. Papageorgiou, I. Papamichail, and I. Nikolos, "Overview and analysis of Vehicle Automation and Communication Systems from a motorway traffic management perspective", Transportation Research Part A: Policy and Practice, vol. 75, pp. 147-165, May 2015.

[2] K. Zhang and S. Batterman, "Air pollution and health risks due to vehicle traffic", Science of the Total Environment, vol. 450-451, pp. 307-316, Apr. 2013.

[3] A. Hegyi, B. De Schutter, and J. Hellendoorn, "Optimal coordination of variable speed limits to suppress shock waves", IEEE Transactions on Intelligent Transportation Systems, vol. 6, no. 1, pp. 102-112, Mar. 2005.

[4] M. Papageorgiou and A. Kotsialos, "Freeway ramp metering: an overview", IEEE Transactions on Intelligent Transportation Systems, vol. 3, no. 4, pp. 271-281, Dec. 2002.

[5] A. Talebpour and H. S. Mahmassani, "Influence of connected and autonomous vehicles on traffic flow stability and throughput", Transportation Research Part C: Emerging Technologies, vol. 71, pp. 143-163, Oct. 2016.

[6] R. E. Stern, S. Cui, M. L. Delle Monache, et al., "Dissipation of stop-and-go waves via control of autonomous vehicles: Field experiments", Transportation Research Part C: Emerging Technologies, vol. 89, pp. 205-221, Apr. 2018.

[7] I. Papamichail, N. Bekiaris-Liberis, A. I. Delis, et al., "Motorway traffic flow modelling, estimation and control with vehicle automation and communication systems", Annual Reviews in Control, vol. 48, pp. 325346, Jan. 2019.

[8] C. Chalons, M. L. Delle Monache, and P. Goatin, "A conservative scheme for non-classical solutions to a strongly coupled PDE-ODE problem", Interfaces and Free Boundaries, vol. 19, no. 4, pp. 553-570, Jan. 2018.

[9] C. Lattanzio, A. Maurizi, and B. Piccoli, "Moving Bottlenecks in Car Traffic Flow: A PDE-ODE Coupled Model", SIAM Journal on Applied Mathematics, Society for Industrial and Applied Mathematics, vol. 43, pp. 50-67, 2011.
[10] G. Piacentini, P. Goatin, and A. Ferrara, "Traffic control via moving bottleneck of coordinated vehicles", Proceedings of 15th IFAC Symposium on Control in Transportation Systems, vol. 51, no. 9, Jan. 2018.

[11] M. Cicic and K. H. Johansson, "Traffic regulation via individually controlled automated vehicles: a cell transmission model approach", in 21st International Conference on Intelligent Transportation Systems (ITSC), IEEE, Nov. 2018, pp. 766-771.

[12] C. F. Daganzo, "The cell transmission model: A dynamic representation of highway traffic consistent with the hydrodynamic theory", Transportation Research Part B: Methodological, vol. 28, no. 4, pp. 269-287, Aug. 1994.

[13] A. Vahidi and A. Sciarretta, Energy saving potentials of connected and automated vehicles, Oct. 2018.

[14] M. Zambelli and A. Ferrara, "Robustified distributed model predictive control for coherence and energy efficiency-aware platooning", in 2019 American Control Conference (ACC), Jul. 2019, pp. 527-532.

[15] K. Y. Liang, J. Mårtensson, and K. H. Johansson, "Heavy-Duty Vehicle Platoon Formation for Fuel Efficiency", IEEE Transactions on Intelligent Transportation Systems, vol. 17, no. 4, pp. 1051-1061, Apr. 2016.

[16] G. Piacentini, C. Pasquale, S. Sacone, S. Siri, and A. Ferrara, "Multiple Moving Bottlenecks for Traffic Control in Freeway Systems", Proceedings of the European Control Conference (ECC), Naples, Italy, June, 2019.

[17] G. Piacentini, A. Ferrara, I. Papamichail, and M. Papageorgiou, "Highway Traffic Control with Moving Bottlenecks of Connected and Automated Vehicles for Travel Time Reduction", in Proceedings of 58th Conference on Decision and Control (CDC 2019), 2019.

[18] G. Piacentini, P. Goatin, and A. Ferrara, "A Macroscopic Model for Platooning in Highway Traffic", SIAM Journal on Applied Mathematics, vol. 80, no. 1, pp. 639-656, Jan. 2020.

[19] M. J. Lighthill and G. B. Whitham, "On Kinematic Waves. II. A Theory of Traffic Flow on Long Crowded Roads", Proceedings of the Royal Society of London. Series A., vol. 229, pp. 317-346, 1955.

[20] P. I. Richards, "Shockwaves on the highway", Operations Research, vol. 4, pp. 42-51, 1956.

[21] R. A. Ramadan and B. Seibold, "Traffic Flow Control and Fuel Consumption Reduction via Moving Bottlenecks", 2017.

[22] J. Zhao, R. Zhao, G. Wang, and X. Zhang, "Analysis of fuel economy of autonomous vehicle platoon", in ICTE 2013 - Proceedings of the 4th International Conference on Transportation Engineering, 2013, pp. 980-986.

[23] A. K. Bhoopalam, N. Agatz, and R. Zuidwijk, "Planning of truck platoons: A literature review and directions for future research", Transportation Research Part B: Methodological, vol. 107, pp. 212-228, Jan. 2018.

[24] S. Tsugawa, S. Jeschke, and S. E. Shladover, "A Review of Truck Platooning Projects for Energy Savings", IEEE Transactions on Intelligent Vehicles, vol. 1, no. 1, pp. 68-77, Jun. 2016. 